\title{
Transparency as the Best Policy: Risk Communication for Both Business and Beyond
}

\author{
Gun-Jo Woo' ${ }^{1}$, Hyejung Chang ${ }^{2}$ \\ ${ }^{1}$ Lee International IP \& Law Group, Seoul, Korea \\ ${ }^{2}$ Kyung Hee University, Seoul, Korea
}

\section{The Role of Communication in the Midst of an International Crisis}

Today more than ever do people take risk into account as they venture out into their everyday lives. The current pandemic presents a unique case of risk in that its potential outcomes for individuals and groups are contingent upon a complex interplay of individual and collective behaviors, understandings, and objectives. The communication of risk thus plays an important role in facilitating this intersection over the indefinite span of uncertainty before us as it inevitably grows to affect not only our world but also our understanding of everyday life. One unique feature of such an interplay is that it shows how socially perceived risks tend to have a greater impact on society than objectively evaluated risks. As the perceptions of these risks differ depending on the parties involved (e.g., individuals, organizations, countries), relational networks and the communication that makes them possible take on even more importance.

Since the spread of the coronavirus that originated in China at the end of 2019, the world has been transformed into communities of insecurity, anxiety, and tension. Every day, thou-

Received: Nov 18, 2019 Revised: Dec 17, 2019 Accepted: Dec 23, 2019 Corresponding author: Hyejung Chang

School of Management, Kyung Hee University, 26 Kyungheedae-ro, Dongdaemun-gu, Seoul 02447, Korea

Tel: +82-2-961-9432, E-mail: hjchang@khu.ac.kr

This is an Open Access article distributed under the terms of the Creative Commons Attribution Non-Commercial License (http://creativecommons.org/licenses/ by-nc/4.0/) which permits unrestricted non-commercial use, distribution, and reproduction in any medium, provided the original work is properly cited.

Copyright ( 12020 Korean Association for Business Communication. sands of cases are confirmed, with many of them resulting in death, deepening concern around the world. Experts have said and argued that the spread of this virus is a result of China's problematic communication system. The lack of communication between the Central Communist Party and the country's local government officials prevented an adequate and quick response in the early stages of the outbreak (Fisher, 2020). According to John Yasuda, a specialist on Chinese government at Indiana University, the coronavirus pandemic is comparable to the 2002 outbreak of SARS (Severe Acute Respiratory Syndrome) in terms of the reasons for its spread (Fisher, 2020). The growth of SARS also grew uncontrollably as a result of local officials concealing reports of initial infections, which led to infections worldwide. Local officials in China tend to hide or ignore these crisis situations because they know they would ultimately be blamed for them. It is therefore almost impossible to hear about local problems when they occur, and reports are usually delivered only once the problem has grown significantly. This situation highlights the importance of communication in risk management.

\section{Communication Issues for Risk Management in Business}

The same is true for companies in crisis that face unexpected threats from both inside and outside the organization. Businesses face large and small crises every year, and some of them have seriously undermined their survival. For example, with the recent coronavirus pandemic, the aviation, tourism, and food service industries have taken a significant hit as a result of 
consumer fears while industries relevant to the situation such as medical institutions, mask and sanitizer manufacturers, and pharmaceutical companies producing testing kits are struggling with sudden surges in demand. On a microscopic level, the various crises of individual companies have had a significant impact on society. Events that threaten the safety of consumers, such as humidifier disinfectants, GM (genetically modified) foods, and mad cow disease, remain in the minds of the people long after the crisis (Korea Ministry of Food and Drug Safety, 2016). Here, the success or failure of a company varies greatly depending on how each company facing the crisis communicates with its consumers (Diers-Lawson, 2020).

In general, when a company faces a crisis, it first tries to avoid or hide its problems and then tries to solve them quietly and internally (Kaplan \& Mikes, 2012). The reason given for this response is that the company itself often does not have an accurate analysis of what they are facing, but considered externally, this move is a self-defense mechanism to delay exposure to the public and secure time to think of ways to prevent deterioration of the company's image. Specifically, most companies tend to respond in one of the following ways:

- No response or a delayed response: The company deliberately stays silent due to worry about image loss or delays its response because it is difficult to make decisions due to a lack of a full understanding of the situation.

- Inaccurate communication: To avoid responsibility, the company produces an inaccurate, dampening the gravity of the situation.

- Inconsistent communication: The organization's position is expressed inconsistently by expressing intentions from different positions due to a lack of consensus among the organization's members.

- Unstructured risk management system: The goals of risk management are not clear, so the crisis situation cannot be managed systematically.

- Potential of continuous crisis: As the company lacks a process to improve the problem by diagnosing and evaluating the progress after the crisis is over, the organization continues to have potential risk factors that can repeatedly cause a crisis.

Failure to respond promptly and appropriately when a crisis occurs may resolve it in the short term. However, in the long term, these communication mistakes result in lost trust with various stakeholders due to the spread of inaccurate or distorted information. Companies thus need to respond to crises with an immediate, proactive and transparent communication strategy that reduces the hiding or avoidance of the problem.

\section{Considerations for Proactive Risk Communication}

\section{Advent of New Channels of Communication}

With the development of information technology, communication patterns between individuals are expanding with channels such as text messaging (SMS, MMS), Kakao Talk, WhatsApp, Facebook, Twitter, and Instagram. In addition to these additional communication channels, the ways of taking in the world are changing as lectures in the classroom are now provided online through e-learning platforms such as TED and YouTube. These new technologies are rapidly changing the way we communicate, making them an important factor to consider in a company's risk communication strategy. In particular, the role of non-face-to-face communication is increasing in global risk situations, as can be seen in the current pandemic. These changes bring a variety of strengths as they make possible a form of faster and more practical communication, but they also present shortcomings compared to face-to-face communication channels.

Peter F. Drucker said, "The most important thing in communication is to hear what isn' $t$ being said" (Moyers, 1988). In conversation, non-verbal communication channels such as facial expressions and movements play a significant role in conveying meaning. However, as instant messages, e-mails, online lectures, etc. are not observed, there is no additional information that allows us to confirm that the communication was successful. Additionally, when considering the three behavioral factors of communication proposed by a cognitive psychologist David Olson (Olson, 1970, 1977) - utterance, internal meaning in speech, and the mediating action of speech - the new communication channel includes only the formal part of the conversation and has no internal meaning and implication. As a result, the new communication channel may leave the speaker's intention unclear.

Therefore, in the new communication channel of information technology, risk management communication carries the danger of quickly spreading inaccurate or distorted information; this new channel allows for easy and quick communication regardless of place while also allowing for the possibility of content delivery being negatively affected. Therefore, while the advantages of new technology should be utilized, risk communication strategies should be pursued so that technology does not interfere with communication.

Expansion of a "Untact" Culture that is Worried about the Deterioration of Communication

With the recent growth of businesses adapting to consumer payment preferences, cash-free institutions are becoming more 
and more common. Many customers nowadays prefer to pay with their credit or debit cards, not with cash. One trend in this line is consumers installing a restaurant app that allows them to order and pay for their meal all from their mobile phones; all they have to do is go to the restaurant and pick up their food. Various companies are also developing robots that make and serve food, with the ultimate direction being aimed at a process that requires no human intervention. A future in which customers can eat a meal in a restaurant without interacting with a person is not far off.

Korea is one indicator of how far we are from this future. Convenience stores, cafes, and study centers are venues that are often unstaffed. This change is an embodiment of the "untact culture" that characterizes the current millennial generation (i.e., those born in the 1990s and early 2000s). "Untact" is a combination of the English word "contact" and the negating prefix "un." It refers to a culture in which services are provided with little to no human contact. A recent report indicates that $87 \%$ of people in their 20s feel more comfortable with a machine than with humans (Kim, 2017). The millennial generation, the mainstay of our society's future, is already ushering in untact culture with and the effects that it will bring.

The spread of untact culture means a reduction in communication. There is also concern that the communicative disconnection will deepen as a result of consumer preference of machines to people, weakening the resistance to conflict found in human relations. This tendency can make more serious the vulnerabilities of communication, such as not reporting or avoiding communication or responsibility in a crisis situation. It is thus necessary to develop a risk communication strategy for future generations who prefer an untact culture.

\section{Use of Al in Risk Communication}

Artificial intelligence (AI), which has been greatly developed in recent years, can change the behavior of communication for crisis management. Specifically, with the development of AI, machine learning technology that can improve communication for crisis management is currently being explored. The field of risk communication applying $\mathrm{AI}$ is largely divided into two areas: (1) predicting and monitoring for early detection and (2) information extraction and classification for situation awareness (Ogie, Rho, \& Clarke, 2018). It is expected that these AI applications will be able to play a broader role by using science based evidence to reduce uncertain systematic factors in a crisis situation.

Since AI relies on collected data to derive meaningful insight, it is important to collect timely and reliable data. Fortunately, the AI algorithm not only analyzes a wide range of accumulated data using data mining technology but also enables the ability to provide real-time analysis results by monitoring the reactions and situational changes of stakeholders related to the crisis. The potential of this AI can be valuable in providing a clear communication direction in crisis situations.

Furthermore, with the development of AI, it is possible to provide direct services by robots. Social activities that were conducted offline to adapt to a crisis situation such as the coronavirus are changed to face-to-face video conferencing, online lectures, online interviews, and online communication. In a crisis situation, the customer center service that consults face-to-face with the consumer can also secure a competitive advantage in a new era by using an AI robot as a substitute for human beings, taking part in communication with the consumer. Robots are in charge of normal communication, and organization members can focus on more strategic roles in crisis situations to improve the efficiency and effectiveness of transparent communication in order to overcome a crisis.

\section{Direction of Risk Communication Responding to Unpredictable Risks}

Unexpected new types of risk emergence, and various risks threaten our future lives. Some of the risks are predictable and some are impossible to predict, but in recent years, unpredictable risks tend to be increasing (Rogers \& Feiss, 1998). Since these risks do not simply stay in one location, but affect the entire world, the risks have become a social phenomenon that humankind must consider and solve together. Therefore, the risk communication of a company may remain a more important issue than how the risk is perceived by stakeholders because the risks can be developed in a serious situation that determines the life and death of the company.

Effective communication in a crisis situation plays an important role in guiding stakeholders as they respond. The cause of the risk itself may not be in the realm of communication, but in physical, biological, mechanical, etc., area. However, the process of early detection of an immediate risk, finding a solution, and solving a problem is an extremely social task. In responding to crisis, the results differ greatly depending on the culture, law, system, and environment, whether at the national level or the corporate level. Therefore, organizations seeking solutions by choosing alternatives with a high probability of success in the midst of a wide range of uncertainties in which the crisis can progress will be able to resolve the crisis as quickly as possible.

The risk factors of companies are emerging more rapidly and seriously, thanks to the development of new communication channels such as SNS and the spread of the untact culture, and 
this trend is increasing (Sharma \& Verma, 2018). Therefore, if the occurrence of a crisis is inevitable, companies should also build a communication system that can respond quickly and proactively with expertise and the continuous monitoring in preparation for risk from the overall perspective of the organization. A systemic approach using recently developed $\mathrm{AI}$ also seems likely to help build a crisis management communication system involving multifaceted stakeholders.

In conclusion, since companies are exposed to various unpredictable crisis situations, it is important to implement an honest and transparent communication strategy based on a pre-established integrated crisis management system when a crisis occurs. A company's effective communication strategy should approach all members through a system built at the structural level of the company to enable shared and consistent communication. There is a Korean proverb saying, "use a spade to block something that a mere hoe could block," which means something similar to "one stitch in time saves nine" in English proverb. These proverbs mean that it cannot be solved when it is a small thing and that it is hard to stop after it gets bigger and more serious. It is not uncommon to try to hide a crisis situation that can be solved relatively easily before the situation becomes serious, and delaying disclosure will eventually make it difficult or impossible to resolve it even with great cost and effort. Therefore, science and data based transparency is the best policy in business communication for risk management especially in unpredictable future environment.

\section{References}

Diers-Lawson, A. (2020). Crisis communication: Managing stakeholder relationships. New York, NY: Routledge.

Fisher, M. (2020, January 25). Coronavirus exposes core flaws, and few strengths, in China's governance. The New York Times. Retrieved from https://www.nytimes.com/2020/01/25/world/asia/ coronavirus-crisis-china-response.html

Kaplan, R. S., \& Mikes, A. (2012, June). Managing risks: A new framework. Harvard Business Review. Retrieved from https://hbr. org/2012/06/managing-risks-a-new-framework

Kim, Y. K. (2017, April 5). Kiosk speeding up the unmanned trend. Weekely Technology Trend. Retrieved from https://www. itfind.or.kr/publication/regular/weeklytrend/weekly/list. do?selectedId $=968$

Korea Ministry of Food and Drug Safety. (2016). 2016 Ministry of Food and Drug Safety white paper (Reg. No. 11-147000-00190201). Cheongju, Korea: Author.

Moyers, B. (1988, November 17). Peter Drucker: Father of modern management. Retrieved from https://billmoyers.com/content/ peter-drucker/

Ogie, R. I., Rho, J. C., \& Clarke, R. J. (2018). Artificial intelligence in disaster risk communication: A systematic literature review. Paper presented at the 5th International Conference on Information and Communication Technologies for Disaster Management (ICT-DM), Sendai, Japan.

Olson, D. R. (1970). Language and thought: Aspects of a cognitive theory of semantics. Psychological Review, 77(4), 257-273.

Olson, D. R. (1977). From utterance to text: The bias of language in speech and writing. Harvard Educational Review, 47(3), 257-281.

Rogers, J. J. W., \& Feiss, P. G. (1998). People and the earth: Basic issues in the sustainability of resources and environment. Cambridge, UK: Cambridge University Press.

Sharma, S., \& Verma, H. V. (2018). Social media marketing: Evolution and change. In G. Heggde, \& G. Shainesh (Eds.), Social media marketing: Emerging concepts and applications (pp. 19-36). Singapore: Palgrave Macmillan. 
